\section{Intersections}

Canadian Journal of Music

Revue canadienne de musique
Intersections CANADIAN JOURAL OF MUSIC
REVUE CANADIENEE DE MUSIOUH

\title{
La Baladodiffusion ou l'écoute comme surgissement du présent
}

\section{Christine Esclapez}

Volume 34, numéro 1-2, 2014

URI : https://id.erudit.org/iderudit/1030871ar

DOI : https://doi.org/10.7202/1030871ar

Aller au sommaire du numéro

\section{Éditeur(s)}

Canadian University Music Society / Société de musique des universités canadiennes

\section{ISSN}

1911-0146 (imprimé)

1918-512X (numérique)

Découvrir la revue

Citer cet article

Esclapez, C. (2014). La Baladodiffusion ou l'écoute comme surgissement du présent. Intersections, 34(1-2), 91-111. https://doi.org/10.7202/1030871ar

\section{Résumé de l'article}

Dans son article " L'enjeu de la philologie », Denis Thouard ausculte ce qu'il appelle la culture post-livresque, c'est-à-dire notre rapport actuel à l'écrit qui se concrétise par une intimité de plus en plus grande avec l'hypertexte.

C'est à partir de ce constat que nous chercherons à ausculter les conditions actuelles de diffusion, principalement caractérisées par le principe de la baladodiffusion (ou podcasting) qui interroge directement la question économique mais aussi esthétique de la réception musicale. Loin de dresser un bilan totalement négatif de ces nouvelles conduites d'écoute qui ont tendance à soumettre la qualité (choix de l'interprétation par exemple) à la quantité des téléchargements musicaux effectués, nous en discuterons de la façon la plus ouverte et la plus utopique possible. Nous ferons l'hypothèse qu'esthétiquement, il y a là, quelque chose d'extrêmement intéressant qui est en train de repousser imperceptiblement les limites de la sphère économique et même politique. Face à cette envie portative, immédiate, relative et désordonnée de musiques plurielles, il y a aussi un renversement des valeurs qui sont les nôtres : la quantité de musiques écoutées n'est plus nécessairement synonyme d'appauvrissement, d'inculture ou d'asservissement aux logiques de l'économie de marché, mais procède aussi de ce désir de découvrir et de tendre l'oreille. Désir où le total désordre stylistique et chronologique permet de refonder cette continuité historique mais aussi anthropologique qui semblait avoir déserté la seconde moitié du $\mathrm{XX}^{\mathrm{e}}$ siècle.
Copyright @ Canadian University Music Society / Société de musique des universités canadiennes, 2015
Ce document est protégé par la loi sur le droit d'auteur. L’utilisation des services d'Érudit (y compris la reproduction) est assujettie à sa politique d'utilisation que vous pouvez consulter en ligne.

https://apropos.erudit.org/fr/usagers/politique-dutilisation/ 


\title{
LA BALADODIFFUSION OU L'ÉCOUTE COMME SURGISSEMENT DU PRÉSENT
}

\author{
Christine Esclapez, Aix-Marseille Université, LESA EA 3274
}

Un homme peut, s'il est vraiment sage, jouir, sur une chaise de tout le spectacle du monde, sans savoir lire, sans parler à personne, en n'utilisant que ses sens, à la condition que son âme ne soit jamais triste.

(Fernando Pessoa, Fragments d'un voyage immobile, 51$)^{1}$

\section{D'UN LIEU À L'AUTRE}

Dans son article «L'enjeu de la philologie ${ }^{2} »$, Denis Thouard ausculte ce qu'il appelle la culture post-livresque c'est-à-dire notre rapport actuel à l'écrit qui se concrétise par une intimité de plus en plus grande avec l'hypertexte :

L'hypertexte pris comme le format standard de diffusion de l'information sur le réseau mondial, des passages toujours plus nombreux et souvent arbitraires entre notions sont créés, permettant de sauter avec une grande facilité d'une plage à une autre, pourvu qu'un élément de contiguité, un «lien» y invite le lecteur. Celui-ci se voit ainsi épargner la peine de produire des associations d'idées ou de guider lui-même dans sa recherche : elle se constitue d'elle-même sous ses yeux. (2003, p. 359)

\section{Le constat n'est pas des plus optimistes}

Le passage instantané d'un lieu à l'autre permettant une contiguïté et une association linéaire d'éléments crée un voisinage et une proximité que ces éléments ne partagent pas nécessairement sur le plan thématique, générique ou même historique. L'autonomie du lecteur est comme grignotée par la standardisation du format et le principe de l'open link: «l'établissement d'une cohérence locale et la confrontation de différents textes est rendue improbable ${ }^{3}$. Cette recherche qui se constitue d'elle-même et qui échappe au lecteur est largement soumise à la mondialisation de la diffusion de l'information que nous vivons depuis les années 9o, c'est-à-dire qu'elle s'uniformise progressivement mais sûrement. Elle est, de plus, totalement dématérialisée dans la mesure où le livre, comme objet où le texte s'épanouit et se donne à lire, existe de façon totalement ambiguë. L'hypertexte l'éloigne du lecteur qui n'en prend conscience qu'en

1 Pessoa 1990, p. 65.

Thouard mai 2003 , p. 346-359.

3 Ibid., p. 359. 
transparence, un peu comme s'il devenait une entité immatérielle ou même une pure idéalité, habitant déjà d'un monde lointain et légèrement obsolescent. L'existence de l'objet-livre n'est plus soumise à ses conditions matérielles d'objet concret dans le monde. En d'autres termes, sa prise en main, ses pages feuilletées une à une, ses rythmes de lecture (de la plus lente à la plus rapide, ou à celle en diagonale et oblique) se prêtent à une transfiguration digne des plus célèbres expériences des alchimistes des siècles passés : "Alors que la lecture du livre est placée sous le signe de la durée et d'une certaine continuité, celle de l'hypertexte est caractérisée par un sentiment d'urgence, de discontinuité et de choix à effectuer constamment ${ }^{4}$ ». L'open link ouvre la lecture sur une quantité d'informations qui n'offre pas cependant la certitude d'une extension proportionnelle des connaissances. La lecture et l'objet-livre sont en péril, de même que le maniement de la langue française. C'est bien à une culture autre que nous avons affaire, étrangère à celle qui a été le mode dominant de transmission et d'acquisition des connaissances depuis l'invention de l'imprimerie typographique en Europe par Guttenberg 5 :

Jusque vers la fin des années soixante-dix, on pouvait encore croire que l'ordinateur n'aurait d'effet que sur les domaines scientifiques et techniques. On se rend compte aujourd'hui que cet appareil et les technologies qui l'accompagnent sont en train de révolutionner la façon même dont notre civilisation crée, emmagasine et transmet le savoir. À terme, cette mutation influencera l'outil le plus précieux que l'homme ait inventé pour construire ses connaissances et élaborer son image de soi et du monde : le texte. Et comme celui-ci n'existe qu'en fonction de la lecture, les mutations du premier ont des répercussions sur la seconde, de même que celles de la seconde entraînent nécessairement la mise en place d'autres modes de textualité. On ne lit pas un hypertexte comme on lit un roman, et la navigation sur le Web procure une expérience différente de la lecture d'un livre ou du journal ${ }^{6}$.

Cette réalité est-elle «en mesure de produire l'équivalent d'une philolo$\mathrm{gie}^{7}$ ? » se demandera Denis Thouard dès le tout début de son propos. Car, c'est bien ce nouveau mode de transmission du savoir que devra prendre en compte la philologie des temps futurs sous peine de se voir définitivement rangée dans la rubrique des introuvables. Dans cet article, et sans pour cela se sentir tout à fait rassuré quant à l'avenir de cette future philologie, Denis Thouard préfèrera occulter toute nostalgie (comme celle que nous avons brièvement tracée plus haut) et proposera d'ancrer nos regards dans le monde tel qu'il est en train de changer et de se transformer avec un mot d'ordre à peine voilé : confrontonsnous à notre contemporanéité. L'enjeu de la philologie des temps futurs, comme celle des temps anciens, est de ne pas séparer la lecture des textes du sens

4 Vandendorpe 1999, p. 11. (Voir particulièrement, p. 203-251).

5 Rappelons tout de même qu'en Chine et en Corée, les caractères mobiles en argile ou métalliques similaires à ceux que Guttenberg utilise au $\mathrm{XV}^{\mathrm{e}}$ siècle avaient été inventés plus tôt, dès le $\mathrm{XI}^{\mathrm{e}}$ siècle.

6 Vandendorpe 1999, p. 9.

7 Ibid., p. 350. Voir Rastier 2001. 
de l'histoire et du désir de comprendre et d'apprendre qui constitue la société humaine, même si pour cela elle doit accepter de se décadrer et de revisiter certains des modes de représentation qui ont été les siens depuis longtemps ${ }^{8}$. Pratiquer la remise en cause comme une seconde peau en se confrontant à ce qui lui est in-compréhensible (qui n'a pas encore de forme et de contours précis) et qu'il ne s'agit peut-être pas de rejeter d'emblée ou de qualifier hâtivement de dégénérescence irréversible de la société humaine. Cette seconde peau n'est pas une seconde vie (Second Life) toute frémissante du risque schizophrénique. Si le principe même d'un jeu comme Second Life est de construire un monde multiple qui évolue en dehors même du participant au jeu ${ }^{9}$ puisqu'il est le résultat de la ramification des actions des autres participants situés un peu partout dans le monde réel, la seconde peau que nous évoquons pour tenter de comprendre l'enjeu de la philologie serait, à l'inverse de ce processus, la condition même de la connaissance. Défense passionnée d'une certaine forme de liberté critique. Liberté que s'octroie celui qui tente de construire le monde à partir de son interprétation des textes en provoquant des rencontres intertextuelles (culturelles, historiques ou même esthétiques) et parfois même anachroniques. Seconde peau parce qu'elle qualifie ce processus comme faisant corps avec la volonté, la sincérité, la culture et la curiosité de celui qui aborde les textes pour se comprendre en même temps qu'il tente de comprendre autrui.

Les profondes mutations qui touchent nos domaines d'acquisition des connaissances interrogent fortement les conditions mêmes de l'acquisition de ces connaissances. Si elles paraissent les remettre en cause fortement au point de les dissoudre en les éloignant du sujet connaisseur, il s'agit ici de prendre le problème à bras le corps en lui insufflant une petite dose d'utopie pour le désenclaver des scénarios catastrophes qu'il semble lui-même créer ou que la doxa semble immanquablement lui prêter. Les médias (radio, blogs, sites dédiés, ouvrages) abordent de plus en plus fréquemment la question et force est de reconnaitre que l'on retrouve bien souvent dans leurs propos une opposition de points de vue digne des plus célèbres querelles entre anciens et modernes qui ont rythmé notre histoire occidentale des idées et des œuvres. On a pu, par exemple, entendre dire que même si la virtuosité dans le maniement des outils de communication actuels reste inégalée (le succès des réseaux sociaux en est, faut-il le rappeler, la preuve évidente), elle n'est pas nécessairement le gage d'un accroissement du partage sensible des expériences, ni même une expression

8 D'ailleurs, dans son article, Claude Coste montre que le livre a encore de beaux jours devant lui : chaque support (livre ou site internet) correspondant à un usage particulier. (Coste 15 décembre 2009).

9 «(...) un jeu comme Second Life exploite un ensemble de caractéristiques, dont celle particulièrement efficace, de persistance. Que veut dire et à quoi renvoie le terme "persistance»? Il signifie que le jeu se développe continûment avec ou sans la présence du joueur : le jeu en entier se comporte comme le monde qui nous entoure, ce monde naturel qui vit croit et meure sans nous. Ainsi, quand un joueur (mais disons plutôt un participant) quitte Second Life, le monde de Second Life continue à croître en son absence, et quand ce même participant y revient, il s'aperçoit que l'arbre qu'il a planté la veille sur son terrain a été détruit, mais que ses haricots ont poussé... Il s'agit là d'un environnement ouvert, et non d'une mise en scène dans les limites de décors préfabriqués. Cet environnement a la capacité d'être en transformation continuelle sous l'action de tous les participants (...).» (Cauquelin 2010, p. 158-159). 
accrue des émotions. Pour certains, cette communication dématérialisée crée une carence expérientielle qui ne parvient plus à s'approprier le live : la vie en train d'être vécue. Ces constats dont nous avons déjà mis en évidence la relative justesse nous souhaitons les désenclaver des prévisions millénaristes qui les accompagnent inévitablement, tout en refusant d'être les dupes d'une logique mercantile désormais mondialisée que Jean-François Lyotard supposait, dès la fin des années 70, dans son Rapport sur le savoir dans les sociétés les plus développées proposé au Conseil des Universités, auprès du gouvernement du Québec :

Avec l'hégémonie de l'informatique, c'est une certaine logique qui s'impose, et donc un ensemble de prescriptions portant sur les énoncés acceptés comme "de savoir». On peut dès lors s'attendre à une forte mise en extériorité du savoir par rapport au "sachant», à quelque point que celui-ci se trouve dans le procès de connaissance. L'ancien principe que l'acquisition du savoir est indissociable de la formation (Bildung) de l'esprit, et même de la personne, tombe et tombera davantage en désuétude. Ce rapport des fournisseurs et des usagers de la connaissance avec celle-ci tend et tendra à revêtir la forme que les producteurs et les consommateurs de marchandises ont avec ces dernières, c'est-à-dire la forme valeur. Le savoir est et sera produit pour être vendu, et il est et sera consommé pour être valorisé dans une nouvelle production : dans les deux cas, pour être échangé. Il cesse d'être à lui-même sa propre fin, il perd «sa valeur d'usage ${ }^{10}$ ».

\section{Pourtant...}

Les ruptures et les mutations nous confrontent au changement, à l'inconnu (et avec lui à la peur) où ce qui est en train d'être vécu ne parvient pas à visualiser et à imaginer ce qui est devant lui, encore en advention ${ }^{11}$. De ce qui est en advention, combien il est difficile de prévoir la postériorité alors qu'il est encore in-forme : corps étranger, chose inutile et gênante qui vient bousculer les valeurs collectives de la culture dominante, elles-mêmes souvent acquises après de longs labeurs et contraintes, par la force des choses, à être amnésiques à ce qui a été leur passé. Marche zigzagante de l'histoire des idées et de leur invention que celle qui rythme nos modes de représentation et d'acquisition des connaissances. L'historienne Colette Sirat remarque :

Il faudra vingt siècles pour que l'on se rende compte que l'importance primordiale du codex pour notre civilisation a été de permettre la lecture sélective et non pas continue, contribuant ainsi à l'élaboration de structures mentales où le texte est dissocié de la parole et de son rythme. ${ }^{12}$

Ce sera donc à la démarche philologique de s'adapter à cette actualité du texte (dans son acception la plus large de production humaine) qui mêle les dimensions esthétiques à des dimensions marchandes et standardisées et qui pose

\footnotetext{
10 Lyotard 1979, p. 13-14.

11 Voir à ce sujet Esclapez 2003, p. 61-80.

12 Citée par Vandendorpe 1999, p. 53.
} 
avec urgence la question de sa valeur esthétique. Pour Denis Thouard présentant le combat de Jean Bollack pour constituer une philologie critique moderne, c'est bien cette mutation que doivent accomplir tous ceux pour qui le travail sur le sens demeure une chose primordiale. Le sens, vocable ambigu s'il n'est ancré dans la réalité de l'objet ou du territoire qu'il tente d'observer, n'est pas de façon a priori, pas plus qu'il n'est une entité abstraite. Tout au moins en ferons-nous l'hypothèse pour le désenclaver d'éventuelles tentatives de sacralisation qui, de tout temps, ont pu n'y voir que la légitimation de leurs propres idéologies ou régimes de pensée. L'élargir au contact de l'actualité, tout en prenant garde à ne pas sombrer dans les effets de mode ou à ne pas verser dans un engouement naïf pour le nouveau ainsi observé, reviendrait à tenter d'instaurer un contact avec ce qui, à un moment donné, ne nous regarde plus vraiment et qui pourtant, avec quelque raison, regarde d'autres que nous. Difficulté de la remise en question qui finalement n'est pas uniquement une question de conscience mais bien davantage une question de spatialisation historique car elle est celle du passage générationnel ${ }^{13}$ qui fait de nos us et coutumes, de nos façons de voir et de concevoir le monde et sa réalité, de notre propension à nous situer par rapport aux autres et aux déterminations concrètes (sociales, institutionnelles...) qui participent à notre identité en tant qu'être situé dans le monde des réalités obsolètes, vieillissantes, déconnectées, has been... Sauf que les générations passent, et en passant s'approprient chacune à leur manière le monde tel qu'il va, tel qu'il leur est imposé et tel qu'elles le construisent en le pensant et en l'utilisant. La génération Y, communément nommée Génération Peter Pan née entre le début des années 70 et la fin des années 90 est particulièrement concernée par cette standardisation du net et cette mutation des outils d'acquisition des connaissances. Elle était encore très jeune lors de l'introduction massive de l'informatique grand public et de l'électronique portable (téléphonie mobile, photo numérique, GPS...). La maitrise intuitive que cette génération a acquise de ces outils lui permet de vivre cette révolution technologique comme une fenêtre identitaire pour appréhender et comprendre le monde. Dans le même temps, cette popularisation d'internet s'est elle-même accompagnée d'une culture mercantile et d'une économie des contenus largement tributaire des économies des marchés financiers internationaux. Si la génération précédente, celle des années 60 et 70 (génération $\mathrm{X}$ ) est une génération transitoire, nomade, en quête d'elle-même ${ }^{14}$, la génération $\mathrm{Y}$ intègre ce qui est une révolution technologique comme partie intégrante de son horizon de pensée et d'action. Elle vit le monde globalisé et mondialisé par les économies de marchés comme une évidence dans l'oubli quelquefois de son histoire et de ses souffrances et sa virtualité comme une autre forme de réalité. Depuis les années 2000, le renforcement de ces logiques s'est accru : le web collaboratif (dit Web 2.0 ou Web

13 Nous employons ce terme de génération dans son acception démographique où il fait l'objet d'un consensus, tout en sachant que le lien entre appartenance générationnelle et comportement est sujet à controverse, à discuter et certainement à envisager dans un rapport autre que celui d'une relation de cause à effet.

14 Cette génération a connu les plus grands bouleversements de mentalités qui ont traversé le $\mathrm{XX}^{\mathrm{e}}$ siècle comme la Chute du mur de Berlin. 
social) ${ }^{15}$ détermine une nouvelle étape de cette évolution technologique sans que l'on ne puisse prévoir ce que donnera cette interactivité généralisée sur la toile tant du point de vue humain que culturel. La génération qui vit avec cette expansion, prémisse d'une mutation politique et idéologique, n'est plus réellement la génération $\mathrm{Y}$, mais ce que l'on nomme (depuis 2005) la génération $\mathrm{Z}$ ou génération silencieuse. Génération dont on ne sait pas encore ce qu'elle sera, ni ce qu'elle fera. Notamment parce que la part de "création» dans la diffusion et la production de données sur cette «toile d'araignée mondiale» que sont le web et l'internet remplace progressivement leur simple utilisation. En février 2010, Luc Chatel, Ministre de l'éducation nationale française, a fait part des conclusions du rapport sur le développement du numérique dans l'enseignement scolaire rédigé par Jean-Michel Fourgons : Réussir l'école numérique ${ }^{16}$. Il semble que l'heure de la généralisation du numérique dans l'école française ait sonné, développement qui devrait permettre de combler le retard français en la matière et de façon plus profonde, de favoriser un nouveau rapport à la connaissance et à l'acquisition des connaissances : développer un sens critique et permettre par la pratique créatrice une plus grande autonomie d'action et de pensée. Cette école numérique est, quoiqu'il en soit, le futur de la génération $\mathrm{Z}$ avec laquelle, il est nécessaire de garder le contact pour demeurer au plus près de la modernité agissante. Pour continuer obstinément à apposer une seconde peau sur les zones d'ombres que nos idéologies personnelles et culturelles ne nous permettent pas toujours d'entrevoir sereinement. C'est ainsi que pour Denis Thouard :

On comprendrait alors le lien entre la philologie et la modernité, si fortement soulignées dans La Grèce de personne ${ }^{17}$. Le philologue, tel que le portrait s'en dessine ici, ne serait pas l'érudit passionné d'antiquité, l'amoureux du grec, mais un des protagonistes de la modernité, l'accompagnant dans ses plus hauts accomplissements esthétiques. (...) Est-il si sûr que la mutation des supports rende obsolète la recherche obstinée du sens $(\ldots) ?^{18}$

\section{D'un lieu à l'autre... Open the link}

Que de détours nous sont-ils nécessaires pour aborder (enfin) la question énoncée plus haut dans le titre de notre contribution : «La baladodiffusion ou l'écoute comme surgissement du présent». Toute énonciation thématique devrait pouvoir se passer de préalables, de circonvolutions, de traversées hors

15 L'évolution du web est généralement conçue en trois étapes. Le Web 0 est la forme initiale du World Wide Web née en 1994, réservée aux informaticiens et aux spécialistes. Le Web 1 (1995-2004) marque les réels débuts de l'expansion et de la démocratisation de l'internet : e-commerce, médias... La diffusion est dite "verticale» dans la mesure où l'internaute reçoit des informations sans en être l'auteur (internaute "passif»). Enfin, depuis 2005, la version 2.0 marque les réels débuts du web collaboratif (apparitions des blogs, wiki, réseaux sociaux...) et modifie sensiblement les comportements de l'utilisateur qui devient, à son tour, producteur de données.

16 Disponible via http://www.rslnmag.fr/Content/Files/fc/file/rapport-fourgous-chatel-TICE.pdf.

17 Il s'agit de l'ouvrage de Bollack 1997.

18 Thouardp 2003, p. 350. 
de son contexte associé. Ces restrictions, nous nous les adressons à nous-mêmes avec une pensée pour nos lecteurs légèrement perdus, ou même légèrement agacés par ce détournement, voire ce retournement de thématique. Cette conversion semble, a priori, nous éloigner de notre sujet, plus qu'elle ne nous en rapproche. Pourtant, nous sommes bien ici au cour de ce qu'est le principe de l'open link. Principe du passage d'un lieu à l'autre pour tenter de répondre à notre questionnement initial en adoptant implicitement le principe du «lien du sans-lien ${ }^{19}$ » qui est pour Daniel Charles l'équivalent du principe de synchronicité jungien relayé par les postures épistémologiques des théories des transformations :

Comme le dit le neurophysiologiste Karl Pribram, la transformation de Fourier et l'ensemble des procédures mathématiques qui sont à l'œuvre dans notre cerveau ont le pouvoir de «transformer les choses, en partant du monde de l'espace-temps ordinaire où règne la causalité immédiate, en un ordre involué, distribué et non-local, dans lequel des corrélations, et seulement elles, existent.» À ce compte, la synchonicité ne peut que nous sembler bizarre : elle correspond à nos capacités «d'établir un ordre non-local aussi bien qu'un ordre spatio-temporel d'événements»-autrement dit, d'inventer un espace et un temps neufs, et de promouvoir des dimensions esthétiques inédites : de faire advenir ce que Bloch appelle des « utopies concrètes ${ }^{20} »$.

Travailler le domaine de la synchronicité demeure pour Charles essentiel car il permet non seulement de s'extraire de tout système logique qui «ne fait qu'actualiser, par un enchaînement de déductions successives 'les informations contenues implicitement dans les hypothèses ou les axiomes de départ' $(. . .)^{21}$ » mais aussi parce qu'il permet d'inventer et d'anticiper « des solutions à des problèmes 'qui ne se sont pas encore présentés ou n'ont pas encore été résolus'22 ». Glisser de l'hypertexte à la baladodiffusion ne signifie pas leur amalgame mais la mise à jour de corrélations transversales qui permettent d'ausculter ces nouvelles formes d'appropriation des connaissances et du monde que mettent en œuvre les nouvelles générations pour œuvrer, à partir de notre position, à rendre consistant cet ailleurs situé devant nous ${ }^{23}$. Certes, il s'agit ici de revendiquer une posture largement utopique, une utopie concrète au sens où l'entend Daniel Charles via Ernst Bloch car elle nous paraît être la seule façon de conserver comme une seconde peau, la confiance à ce qui nous est étranger. La synchronicité ou corrélation non-locale selon des liens topologiques ou sémantiques

19 Charles 1998, p. 119.

20 Ibid., p. 124.

21 Ibid., p. 125.

22 Ibid., p. 123.

23 Pour Daniel Charles : «La logique ici esquissée présente, on l'a déjà mentionné, des traits communs essentiels avec la «logique du réalisme» élaborée d'autre part en vue d'une «connaissance de l'individuel» par Jean-Claude Piguet. De plus, celui-ci a cru devoir ajouter à la théorie des «totalités internes" une observation à laquelle on ne peut, selon nous, qu'acquiescer : "Le principe fondamental de la logique du réalisme doit être recherché au-delà du Moyen Âge, au-delà des stoïciens et d'Aristote, au-delà de Platon et de Socrate : il faut remonter à la source de la pensée occidentale : en Inde. » Bref, il se pourrait que l'ailleurs fût en réalité situé devant nous.».(op. cit., p. 126). 
d'événements qui ne semblent pas littéralement les contenir ou même être totalement équivalents est risquée. On en conviendra. Ces sauts d'un lien à l'autre naviguent en terrain miné si, par les jeux de langages qu'ils transportent avec eux, la logique et la cohérence ne parvenaient pas être instituées. Ils pourraient cependant s'avérer fertiles pour observer la poétique de la multiplicité ${ }^{24}$ que ces nouvelles formes d'expression semblent inférer.

... Dans la fausse clarté du passé, la vraie et fausse obscurité du présent, cette double fable, le présent est le seul temps du plaisir. Mais ce plaisir est triple, puisque c'est le plaisir du passé, le plaisir du présent, le plaisir de l'avenir. Et comme son lieu majeur est ce que le corps fait au langage, et sa réciproque, ce que le langage fait au corps, il me semble que le comble du plaisir, enfin, un, je ne veux pas compter, de ses moments forts passe par le sens qu'on peut avoir, inséparablement, de ce qui est en train de naître et de ce qui est en train de se défaire dans toutes les formes de prise sur le présent. Sans même que ceux en qui la transformation opère le sachent.

Le plaisir est de s'entraîner à voir, à entendre tout ce qu'on ne sait pas qu'on voit et qu'on entend. C'est pourquoi l'art du langage est le lieu majeur de cette écoute de l'écoute. La constante remise en jeu du sens du langage. Le plaisir d'observer et le tourment d'y être pris autant que prenant. Où se jouent toutes les notions qu'on croit tenir mais qui nous tiennent, et dont il faut se déprendre, sans garantie, si on sait de quoi on se retire. De toute façon, on n'a jamais su d'avance vers quoi on va, et ce qui fait que certains se vident, d'autres tiennent ${ }^{25} \ldots$

\section{LA BALADODIFFUSION, «UNE HISTOIRE DE NOS OREILLES ${ }^{26}$ "}

Le phénomène du podcasting (également nommé baladodiffusion) ${ }^{27}$ est un phénomène récent lié à l'arrivée en 2001 sur le marché culturel de cette petite chose, devenue en peu de temps si précieuse qu'est le iPod, le baladeur numérique créé par Apple qui n’a cessé depuis de se transformer et se démultiplier en plusieurs gammes de produits. De façon parallèle, Apple a créé le logiciel iTunes qui permet le transfert de données vers le iPod qui, dès lors, s'est imposé comme l'outil numérique de pointe intimement lié à l'accroissement du monopole Apple avec le concours de la plateforme commerciale iTunes Store permettant l'achat et le téléchargement de musiques et de nombreux autres contenus (images, vidéo, films...). Le terme de podcasting est ainsi, comme le note Mélanie Millette, un «mot-valise qui résulte de la fusion des termes

24 Charles 1998.

25 Meschonnic 2009, p. 81. Cet extrait est l'introduction d'une section de cet ouvrage particulière intéressante (et dérangeante), «Comment jouir de l'inconnu », p. 81-88.

26 Le titre de cette section est une référence directe à l'ouvrage de Perter Szendy. (Szendy 2001)

27 La distinction entre l'appellation francophone et anglophone est discutée par Mélanie Millette dans son mémoire de maîtrise en communication présenté en 2009 à l'Université du Québec à Montréal : Usages contributifs sur Internet : le podcasting indépendant et le sens de son style. Retenons pour l'instant que l'office québécois de la langue française a adopté en 2005 les mots «baladodiffusion» et «balado» pour traduire les termes «poadcasting» et «podcast» (Millette 2009, p. 8). 
'iPod' (...) et 'broadcasting' qui signifie littéralement 'diffusion' ${ }^{28}$ ». Ce format-phénomène qui symbolise indéniablement notre mode de vie numérique naissant fait l'objet de recherches récentes (et encore peu nombreuses) ${ }^{29}$ dans le champ des media studies ${ }^{30}$. Nées récemment dans le sillage des cultural studies, les media studies s'intéressent aux développements des industries culturelles et de l'internet et observent la pratique des usagers qui utilisent ces nouveaux moyens de communication et d'acquisition des connaissances. Elles partagent, avec les cultural studies, la même volonté d'élargir les sciences humaines aux pratiques populaires ou à celles qui sont en marge des recherches conventionnelles. Dans le champ musicologique, on notera par exemple l'essor des gender studies, cherchant à réhabiliter la place de la création féminine dans l'histoire de la musique occidentale. Ces nouvelles orientations ont commencé à émerger à la fin des années 60 en Angleterre dans le sillage des préoccupations postcoloniales qui ont accepté de regarder la culture de l'autre ainsi que les cultures autres, jusque-là considérées comme minoritaires et jugées peu dignes d'intégrer le champ des recherches musicologiques ${ }^{31}$. S'intéresser dès lors à un phénomène comme la baladodiffusion, c'est élargir le champ de la recherche en musicologie à de nouvelles façons d'ouvrir nos oreilles, même si pour cela nous devons intégrer à notre raisonnement des logiques mercantiles et mondialistes dont nous ne serons pas les dupes. Si le phénomène de la baladodiffusion est pluriel, nous le réduirons ici à l'une de ses facettes : celle de l'écoute, c'est-à-dire le balado ${ }^{2}$ audio (son) et enrichi (son + image). C'est aussi pour cela que nous utiliserons le terme de baladodiffusion et non celui de podcasting dont les recherches de Millette montrent pourtant la pertinence linguistique et l'actualité de l'usage pour les podcasters 33 . Une précision s'impose. Les travaux de la chercheuse canadienne s'intéressent au «podcasting indépendant», c'est-à-dire : «non-institutionnalisé, non-commercial, créé spécifiquement pour une diffusion sur Internet» et considèrent «le podcasting comme résultant de l'initiative d'un usager (ou groupe d'usagers) indépendant, créant ses contenus avec le matériel dont il dispose, de manière privée et non institutionnalisée, loin des moyens de diffusion professionnels et des contraintes commerciales de l'industrie culturelle ${ }^{34}$.» Cette problématique de recherche s'ancre dans les dernières extensions du web collaboratif impliquant dès lors une véritable culture participative où les valeurs d'usage sont la spécificité des contenus construits sur mesure, «faits maison» impliquant une extension

28 Ibid., p. 4.

29 Nous souhaitons remercier Mélanie Millette pour avoir mis spontanément à notre disposition son mémoire de recherche sur le poadcasting indépendant, un des rares travaux de recherche actuels qui pose un large regard sur ce phénomène et en effectue une analyse pertinente et riche : http://grm.uqam.ca/?q=millette. Voir également BULL 2005, p. 343-355. (disponible via http://www informaworld.com).

30 Voir à ce sujet Millette 2009, p. 28-29.

31 Abbate 2004, p. 822-830.

32 Le balado est le fichier (audio ou vidéo) destiné à être téléchargé (vidéoconférence, reportage, émission de radio ou autre).

33 Millette 2009, p. 8-9.

34 Ibid., p. 7. 
de l'utilisation des balados vers la préproduction, l'enregistrement, la postproduction et la publication. En termes de comportement, on notera également une extension du nomadisme des contenus médiatiques, eux-mêmes à l'image de la mobilité de leurs usagers : «l'écoute ou le visionnement d'un contenu se fera au moment opportun pour l'usager et non à celui imposé par la grille horaire d'un média de masse ${ }^{35}$.» En ce qui nous concerne, nous nous situerons plus en amont (chronologiquement et épistémologiquement) et adopterons une définition plus réduite. Liée à l'écoute (balados audio et enrichis), elle sera également limitée à la question du téléchargement de musiques variées et de vidéos, téléchargement effectué la plupart du temps avec spontanéité, dans un désordre stylistique équidistant où les genres les plus divers se côtoient sur des listes de lecture constituées à partir du logiciel itunes, sur des plates-formes de streaming comme Deezer, sur des sites web d'hébergement comme Youtube ou d'autres plates-formes moins institutionnelles. Finalement la question sera moins de savoir si la baladodiffusion permet ou non le surgissement du présent (que chaque génération provoque de fait) mais plus précisément de savoir quel type de surgissement du présent (et de mise en action de l'Histoire) permet cet outil de la culture actuelle. De quelles façons les usagers et les jeunes générations s'approprient les connaissances pour construire leurs références culturelles et réinvestir le passé? Un passé silencieux que les sons des musiques et des styles rendent présent, souvent en dehors de toute chronologie, pratiquant ainsi une certaine forme de synchronicité dont on se rappellera qu'elle permet, malgré la démarche disjonctive qu'elle met en œuvre, la création du sens. La question de l'économie des contenus, du pouvoir des marchés et des monopoles ne sera pas, non plus, au centre de notre questionnement même si la liberté du geste du téléchargement est largement soumise aux disponibilités des contenus, eux-mêmes tributaires des économies de marché. Seule l'écoute instantanée de musiques plurielles sera discutée dans son rapport à l'instant : l'Instant Time que suggère l'appellation du logiciel source : iTunes. Nous garderons, pourtant, les travaux de Millette en ligne de mire car ils montrent qu'il y a dans ces nouveaux territoires à explorer quelque chose d'extrêmement intéressant qui est en train de repousser imperceptiblement les limites de la sphère économique et même politique. Comme le montre Gérôme Guibert, suivant en cela la logique économique de Karl Polany $\mathrm{i}^{36}$, une utilisation alternative de l'économie de marché est tout à fait envisageable dans la mesure où une pluralité de logiques économiques coexistent souvent à un moment donné et que toutes ne sont pas directement soumises à un régime unique ${ }^{37}$. Envisager une pluralité d'usages à partir de la reconnaissance de la complexité des logiques économiques (économie de marché privée, économie non-marchande publi-

35 Ibid., p. 13.

36 Polanyi [1944]1983.

37 Guibert, http://www.cdmc.asso.fr/fr/ressources/conferences/enregistrements/musique_globalisation). Voir de façon générale, le colloque Musique et globalisation organisé du 9 au 11 octobre 2008 par la revue Filigrane. Musique, esthétique, sciences, société, le Centre de documentation de la musique contemporaine, la Cité de la musique et les universités de Montpellier 3 (RIRRA21), Paris 8 (Esthétique, musicologie et créations musicales), Lille 3 (Centre d'étude des arts contemporains) dont la plupart des conférences sont sur le site du CDMC (cf. supra). 
que ou économie non-monétaire basée sur la réciprocité du lien social) permet la concrétisation de l'utopie de la synchronicité que nous avons envisagée jusque-là comme une base méthodologique mais qui pourrait tout aussi bien devenir une grille de lecture pour envisager le monde tel qu'il semble actuellement se présenter à nous. Et ainsi nous permettre de le lire différemment.

\section{En Écoutant...}

Dans son article «No dead air! The iPod and the culture of mobile listening», Michael Bull ${ }^{38}$ observe certains aspects de l'expérience urbaine contemporaine que sous-entend l'utilisation du iPod dans la culture de ce qu'il nomme «l'écoute mobile». Cette écoute mobile permise grâce à la généralisation des ventes du baladeur numérique commercialisé par Apple est, pour le chercheur, le symptôme d'une «solitude accompagnée» induite par les déplacements fréquents et souvent longs que doit accomplir la population des grandes villes pour se rendre, par exemple, sur son lieu de travail 39 . Une des questions principales du texte est ainsi d'observer comment les utilisateurs du iPod «tentent de peupler les espaces dans lesquels ils se déplacent ${ }^{40} »$. Espaces fluides, mobiles, sans identité singulière, lieux de passage et de traverse, les espaces urbains ne facilitent pas la proximité sociale et le contact humain. Par la création d'une sorte de «bulle d'audition privée ${ }^{41}$ » rendue possible par l'extrême portabilité du iPod et par sa capacité de stockage de plus de 10 ooo titres, l'utilisateur peuple le monde extérieur de façon tout à fait paradoxale : en le rendant quasiment silencieux, en l'annulant presque par le repositionnement intra subjectif que lui suggère la proximité qu'il entretient, lors de l'écoute, avec l'en-soi et le pour-soi de sa conscience. En créant des non-espaces (ou espaces virtuels), l'utilisateur écoute le monde à travers sa subjectivité, et rend l'espace urbain conforme à son désir esthétique ${ }^{42}$. L'écoute instantanée de musiques choisies soit en fonction de l'envie et de l'émotion du moment, soit de façon aléatoire représente, comme le souligne Michael Bull, une sorte de voyage biographique qui rythme les différents moments de la journée ${ }^{43}$. Un des utilisateurs interrogés par Bull spécifie qu'en utilisant son baladeur pour aller en classe, il voit les gens comme il le fait quand il regarde un film, c'est-à-dire en spectateur d'une représentation qui se déroule tout autour de lui, sans réelle interaction avec elle, dans un en-dehors distancié. Un autre évoque l'image du tunnel pour décrire la façon dont il appréhende sa réalité quotidienne avec le casque sur les oreilles. Ce qui est ainsi frappant c'est l'intimité sensorielle entre le sonore et le visuel qui est d'ailleurs sur un plan purement esthétique particulièrement intéressante : le sonore habituellement utilisé comme illustration, imitation du visuel ou support des images devient l'enveloppe sonique par laquelle les usagers appréhendent la réalité extérieure. Le musical rythme leurs déplacements et subjectivise

38 Bull 2005.

39 Ibid., p. 343 et sq.

40 Ibid., p. 344.

41 Ibid.

42 Ibid., p. 350.

43 Ibid., p. 349. 
les espaces sociaux et urbains qu'ils traversent. Il devient le point de jonction entre leur monde intérieur et la réalité telle qu'elle se présente à eux : «The iPod, in effect, warms up the spaces of mobile habitation for users ${ }^{44} »$.

Pour Michael Bull :

This structurally determined image of space and time is at odds with iPod users accounts of urban experience. iPod use provides users with their own "unique» regulated soundscape that mediates the experience of whatever space is passed through and regulates the flow of time as they wish. The meaning of the spaces, oftent received as «non-spaces» bu users, is overlain by the mediated space of their very own sonic envelope from which meaning emanates. IPod users choose the manner in which they attend to these spaces, transforming space and time into their own personalised narrative 45 .

De cette expérience proprement privée (au comble même de la privatisation), quelles pourraient en être les défaillances ou même les impasses? Une sorte de fuite hors des espaces réellement habités semble inévitable, comme pour contourner la mobilité réduite qui semble de plus en plus être la réalité des populations urbaines et dont les causes les plus évidentes sont principalement économiques : prix du carburant qui ne cesse d'augmenter, difficulté d'échapper à l'espace urbain (loisirs, consommation...) mais aussi très pragmatiques : transhumances des week-ends souvent rendues désespérantes par les retours bondés du dimanche soir. Envisager la vie quotidienne avec des écouteurs sur les oreilles crée, selon Bull, une forme de sanctuarisation qui met l'individu à l'abri de la saturation ambiante comblée, paradoxalement dans le cas de l'écoute mobile, par un espace sonore dont l'omniprésence pourrait être analysée comme le symptôme de la peur du vide ou du manque. Nous y reviendrons. La recentration, l'individuation, la déconnexion semblent des réponses à cette saturation communicationnelle (messages, écrans, télévision...) ou à cette difficulté sociétale auxquelles est confronté l'Homme contemporain. Nous sommes continuellement bombardés de messages. L’autre n'a jamais été aussi proche, au moins en termes technologiques (développement des réseaux sociaux), en connexion perpétuelle et cette connexion abolit l'espace et la temporalité de chacun pour les dissoudre dans un réseau arborescent quasi-intemporel, quelque part et nulle part en même temps. Le iPod et, de façon plus générale l'écoute mobile, semble suggérer une autre forme d'appropriation du monde urbain et contemporain, dont l'observation nous renseigne, en retour, sur une autre «manière langagière de fluer ${ }^{46}$ ». À nous de choisir si nous souhaitons rester ou pas connectés. Car ce qui nous intéresse n'est-il pas moins le fait de répondre à ces stimuli que de savoir que l'on va peut-être leur répondre? Il y a là comme la promesse d'une autre attitude (même si elle n'est pas partagée par tous) : celle de réactiver constamment ce qui nous parvient (de l'interpréter?) par la possible déconnexion de ce système de connexion permanente.

44 Bull 2005, p. 353.

45 Ibid., p. 351.

46 Michon 2007, p. 63. 
Ainsi la volonté de gérer le silence en construisant des espaces sonores que l'on est seul à entendre, est-elle peut-être une parole que les corps portent en eux avec d'autant plus de force qu'elle marque, face à cette altérité démultipliée, un espace de régulation. D'ailleurs, cette évocation du silence n'est pas une simple ornementation rhétorique, ni une vue de l'esprit dans la mesure où ce silence que nous recherchons tous quand il s'agit de choisir notre voiture, notre maison, notre quartier devient une valeur essentielle, celle de l'absence d'informations non demandées. Espace propice du choix, il est aussi celui du présent retrouvé et élargi. Terreau de l'utopie?

Une autre manière langagière de fluer... Cette expression est employée par Pascal Michon pour creuser la question des rythmes du politique. L'hypothèse de départ est reprise de Marcel Mauss qui «a montré que les corps sont soumis à des formes de mouvement et de repos, des manières de fluer, bref des rythmes, déterminés socialement à travers des «techniques du corps» et définissant ce que nous pouvons appeler une corporéité 47 ». Prolongeant ces premières hypothèses, Pascal Michon creuse la forme temporelle suggérée par l'économie qui est la nôtre depuis la fin des années 90 : le capitalisme mondialisé. La temporalité contemporaine, néo-capitaliste, conduit à une fluidité, à une flexibilité et à une décentralisation qui engendrent une polyrythmie contrainte par un pouvoir tout aussi dominant que les régimes politiques antérieurs mais plus difficile à circonscrire. Une conséquence de ce régime temporel est de menacer l'individu dans ce qu'il a certainement de plus précieux : son caractère ${ }^{48}$ qu'il ne parvient plus à se forger par manque de continuité narrative ${ }^{49}$. On retrouve là le diagnostic de Lyotard : la fin des grands récits qui marque, selon lui, le début de l'attitude postmoderne. Dispersion, équivalence, équidistance ne permettent plus d'envisager la question du progrès et de l'émancipation de l'individu qui avait été au centre des réflexions occidentales, et particulièrement européennes, depuis les Lumières. Se fait ainsi jour un nouveau nomadisme obligatoire (mobilité de carrière, prédilection pour les actions et les objectifs à court terme, relations sociétales épisodiques...) qui n’a plus rien à voir avec l'utopie nomade (subversive) qui était, à la période précédente, le moyen d'échapper au capitalisme centralisé et bureaucratique. Ainsi le capitalisme mondialisé rejoint depuis peu par le capitalisme médiatique appauvrit les contenus, «enjoint de tout dire $5^{50}$ " tout en édulcorant les manières de dire pour permettre à ces informations d'être reproduites et mises en circulation, le plus rapidement possible :

Ainsi les démocraties libérales, qui se voyaient jusque-là comme des machines à produire des individus émancipés, tendent-elles à devenir aujourd'hui d'immenses dispositifs qui assurent, à travers une fluidification généralisée des corporéités, des discursivités et des socialités, la multiplication d'individus faibles et flottants, constamment happés par

47 Ibid., p. 47.

48 Pascal Michon reprend ici les thèses du sociologue et historien américain Richard Sennett (1943-) sur le monde de l'entreprise néo-libérale. (Ibid., p. 289-298).

49 Ibid., p. 287.

50 Ibid., p. 300. 
les besoins de la production et de l'échange marchand et les interactions dans lesquelles ils sont pris ${ }^{51}$.

Ce sont les dernières phrases de l'ouvrage de Pascal Michon. Le tableau est sombre. L'avenir encore plus. Pourtant, les promesses à peine murmurées de cette autre manière langagière de fluer ne nous semblent pas tenues. Certes du point de vue politique et social, l'homme est un produit, au même titre que ses œuvres et ses objets de connaissance, de la société qui l'a vu naître et de son régime temporel. Mais n'est-ce pas faire de l'homme et de la femme des produits idiosyncrasiques? Des produits sans histoire? Cette Histoire n'est pas, celle, causale des historiens mais celle des rénovateurs (Barthes, Foucault, Bloch ou LeGoff) qui, à rebours et inscrite dans la mémoire collective et individuelle des êtres et des sociétés, est réinventée par chaque génération mais aussi cristallisée dans chaque génération. D’ailleurs, Michon fait rapidement référence à ce qui nous semble pouvoir nous permettre de réenvisager la question de la flexibilité du monde actuel sous un angle, plus confiant. Ce réajustement nous permettra de revenir à l'écoute mobile comme surgissement du présent devenu, au fil de nos questionnements, réticulaire. Citant Diderot, Michon rappelle que le rythme bénéfique évoqué par le philosophe (dans la mesure où on le considère différemment du mètre) "possède la capacité de dépasser les oppositions de l'âme et du corps, du singulier et du collectif, de la nature et de la culture, du travail et du jeu, permettant ainsi la production d'une individualité à la fois plus intégrée et plus puissante ${ }^{52}$ ». Dans ce cas-là, le rythme se met au singulier ce qui implique une morphogénèse étymologique, et une petite dose d'utopie et d'idéalisme qui font de cet homme et cette femme contemporains des produits certes flottants mais un peu moins faibles. Séparé du mètre, le rythme devient «la structure d'un système (sonore) organique conçu sous la catégorie du devenir ${ }^{53}$ ». C'est-à-dire que le rythme est la modalité particulière du devenir d'un être, impliquant, tout autant que les contraintes, désir et accroissement, accomplissement, intégration et appropriation. Et cela, non pas en totale déconnexion avec la réalité sociétale, économique ou politique mais avec elle, en même temps qu'elle en tant que l'être est un habitant du temps qu'il rénove tout en l'épousant, qu'il dit en le parlant, qu'il arpente en le vivant tout autant qu'en le rythmant.

J'ai pu m'entretenir avec de jeunes adolescents et adolescentes ${ }^{54}$. Comme d'autres, je me suis rendu compte très rapidement que le baladeur numérique est l'un des objets symboliques (avec l'ordinateur) de leur façon de vivre. Pour tous, leurs musiques doivent accompagner leurs faits et gestes et leur vie ne pourrait être concevable sans cette mobilité musicale. Avec elle, ils intègrent

51 Ibid., p. 307.

52 Michon 2007, p. 298.

53 de Schlœzer [1947]1979, p. 170. Nous avons mis entre parenthèses le terme de sonore dans la mesure où Schlozer définit ici le rythme en musique. Pourtant, nous pensons que sa définition peut acquérir un statut plus global.

54 Nous tenons à préciser que ces entretiens n'ont rien de systématique et que nous ne nous situons pas - et cela de façon totalement assumée - dans le champ disciplinaire d'une sociologie empirique et statistique. 
un autre geste sociétal, devenu monnaie courante : le téléchargement (le plus souvent illégal). Ainsi développent-ils toute une série de stratégies pour contourner la récente loi Hadopi adoptée en 2009 pour préserver la diffusion des œuvres et la protection des droits sur internet. Son évocation ne conduira pas à discuter de son bien-fondé, mais simplement à mettre en évidence les ruses et la perspicacité de ces usagers qui débusquent continuellement sur la toile des sites de téléchargements gratuits. Dans la mesure où les sites gratuits sont progressivement fermés, il s'agit d'être sans cesse en quête d'autres plates-formes de téléchargement dont la découverte se partage entre amis, dans le plus grand secret. Un peu comme les endroits de campagne ou de forêt soigneusement tenus secrets par les chercheurs de truffes, de girolles ou de carpes. S'il ne s'agit pas ici de discuter éthiquement de ce qui ressemble bien à une sorte de désobéissance civile; force est de reconnaître que leur pugnacité n'est pas uniquement soumise à des logiques économiques. Télécharger des musiques en toute gratuité, c'est aussi tenter d'échapper au monopole des contenus détenus par quelques grands opérateurs comme Apple. C’est aussi avoir le sentiment de demeurer indépendants pour conserver à l'écoute sa gratuité, c'est-à-dire sa liberté. « 800 musiques, tu te rends compte, c'est 800 euros» me dira une jeune utilisatrice. Impensable pour leurs petits budgets et en totale contradiction avec la philosophie même de leur utilisation du téléchargement. Télécharger gratuitement ne s'encombre pas de fixation et de conservation de l'information : on ajoute et on retranche à l'envie en fonction de l'évolution de son goût et des modes. La discothèque idéale est malléable et fluide ce qui sous-entend que la valeur d'usage n'est plus celle de l'échange (au sens économique du moins). Glaner dans le flux devient essentiel. C'est d'ailleurs, ce qui fera la différence quand il s'agira de partager ou de publier sur le net leur playlists : opération presque indissociable de l'écoute en temps réel. La plupart du temps, les musiques téléchargées sur iPod sont complétées par le streaming sur Deezer (ou autre). Il s'agit en fait de montrer, à travers ses choix, sa personnalité, sa curiosité ou même son originalité. Car publier, c'est aussi s'exposer et se montrer. Le désir d'engranger des musiques n'est pas uniquement soumis à la tentation ambiante d'une consommation effrénée mais aussi à la découverte de trouvailles qui permettront à l'usager de réaliser le $b u z z$, le bourdonnement, de faire parler, de faire acquiescer, de faire aimer. Pour se rassurer, sentir que l'on est populaire, que l'on a droit à ces moments éphémères de gloire comme Andy Warhol en avait fait la prédiction en 1968 (les 15 minutes de célébrité...). La trouvaille fait partie intégrante de cette déambulation musicale, assumant pleinement que sa valeur a priori infime et aléatoire fonde le savoir. Le plus souvent incités par les consultations journalières sur le net (Deezer, Youtube), dans des journaux spécialisés comme RocknFlok (ou sur leurs sites) ou par des suggestions «d'amis» et "d'amis d'amis», les utilisateurs écoutent ce qui été commercialisé mais aussi l'inédit, l'oublié, l'ancien. En consultant plusieurs listes de téléchargement, le premier constat qui peut être rapidement dressé est que les reprises (qui étaient à la mode, il y a moins de 10 ans) sont dorénavant moins nombreuses que les originaux, que les groupes indépendants côtoient les valeurs sûres du pop ou du rock devenues des classiques : les Doors, Bob 
Marley (grand retour), les Stones, Les Beatles, les Clash. Cela sur fond d'électro, de rap... Certes la qualité sonore n'est pas tout à fait la même qu'à l'heure des bonnes vieilles chaînes stéréo que leurs grands-parents prenaient un soin tout particulier à acheter (avec d'ailleurs le sentiment qu'il s'agissait de l'achat de leur vie). Certes, le titre des albums d'où sont extraits les titres téléchargés n'est plus aussi mythique que pour les générations précédentes. Certes les redécouvertes ne sont pas totalement spontanées et dépendent la plupart du temps des logiques commerciales des grandes majors. Mais de tout cela émane une défense passionnée pour une façon de vivre la liberté, de façon inconsciemment libertaire : on gère l'illégalité avec laquelle on s'arrange, mais on garde un respect pour soi grâce à la possibilité d'effacer des musiques que l'on n'aime plus ou qui ne sont plus en adéquation avec sa propre évolution personnelle. Ainsi le monde subjectivisé qu'évoquait Bull est éminemment plastique et organique. La conception du lieu, le topos mais aussi la chôra, c'est-à-dire le territoire plus large de la cité, est la clé de cette esthétique où il est possible de se balader d'univers en univers, de culture en culture grâce à la polyphonie de sa propre écoute. Le rythme est selon Diderot «l'unité de l'esprit et de la main 55 » que l'on pourrait décliner, à la manière de Roland Barthes dans son cours de 1977 au Collège de France, comme une forme idiorrythmique de cette nouvelle sociabilité de l'écoute :

Au-delà de sa signification religieuse, le mot d'idiorrythmie séduit Barthes par sa capacité à donner une forme verbale au fantasme de sociabilité qui l'habite. Grâce aux vertus de la métaphore, le mot sert de fil conducteur à l'exploitation systématique d'un désir : le rêve d'une vie à la fois solitaire et collective, d'un timing heureux où s'harmonisent le rythme de l'individu et celui de la communautés6.

Barthes montrera la dimension utopique de l'entreprise idiorrythmique où il s'agit somme toute de rassembler sans réduire, de réunir sans confondre : "Comment trouver un projet suffisamment fort pour rapprocher les gens sans s'aliéner à un discours contraignant, qu'il soit politique, religieux ou idéologique?57 ». Ainsi le principal danger d'un régime temporel idiorrythmique est-il le repli sur soi, la solitude, la réclusion volontaire... La peur de la confrontation à la réalité et à son rythme qui pourrait bien venir se superposer de façon contrapuntique à la temporalité vécue de façon rassurante car elle est propre à l'être. L’enveloppe sonique apposée sur le monde extérieur est protectrice, peut-être autarcique. Cela représente certainement un des possibles de son futur : être un palliatif au désenchantement du monde. Une théorie des possibles pourtant incalculable car elle se heurte à l'incertitude du probable. Ce qui adviendra? Nul ne le sait encore. Nous préférons alors tenter de lui donner sens (activité inaliénable de la seconde peau). Celui-ci ne manquera pas d'être utopique, c'est-à-dire qu'il tentera d'imaginer ce qui ne s'est pas encore présenté, ce qui n'est pas encore avéré, mais surtout ce qui, peut-être, verra le jour par

55 Michon 2007, p. 297.

56 Coste 2009, p. 203 [fichier pdf].

57 Ibid., p. 204 [fichier pdf]. 
le simple fait qu'il a été imaginé. Cette utopie-là est concrète (Bloch, Charles); conçue sous la catégorie du devenir. Le point de jonction entre l'écoute hyper subjectivisée et le monde est le vivre ensemble que nous fait espérer l'utopie idiorrythmique : c'est-à-dire sa transformation en une polyphonie dialogique. Le vivre ensemble suppose de s'être extrait de nos rythmes-mètres pour en accepter la fluidité et en favoriser la porosité. Comme l'écrivait Hadrien à Marc :

J'ai rêvé parfois d'élaborer un système de connaissance humaine basé sur l'érotique, une théorie du contact, où le mystère et la dignité d'autrui consisteraient précisément à offrir au Moi ce point d'appui d'un autre monde ${ }^{58}$.

Une information a été annoncée le 3 avril 2008 sur le net qui devait assez rapidement voir le paysage de la musique téléchargée changer : il s'agit de l'alliance de MySpace avec Sony BMG, Universal et Warner. D’une part parce qu'elle introduit dans le système actuel la possibilité de voir iTunes concurrencé de façon tout à fait officielle; d'autre part parce que cette concurrence économique se double d'une ouverture artistique vers la création en donnant la possibilité à de jeunes artistes indépendants (abonnés de MySpace) de pouvoir diffuser et commercialiser leurs créations ${ }^{59}$. Certes, la démarche est loin d'être philanthropique. Mais elle semble émaner d'un diagnostic assez lucide de la réalité des usages individuels du téléchargement adoptés en parallèle aux usages balisés et délimités par les institutions publiques et les entreprises commerciales.

À la question de savoir quel type de surgissement du présent produit l'usage de l'écoute mobile, nous répondrons qu'il s'agit d'un présent déphasé mettant en jeu le décalage de l'écoute mobile et du monde environnant, a priori incompatibles (mais toutefois inséparables). Le déphasage (ou phasing) est un procédé de composition inventé dans les années 60 par le compositeur Terry Reiley et utilisé par Steive Reich dans les années 70 dans le courant des musiques minimalistes. Procédé que le compositeur considèrera comme une forme classique du canon et qui se conçoit comme la superposition de motifs musicaux identiques dont la progressive accélération de l'un par rapport à l'autre crée un

58 Yourcenar [1958] 1974, p. 22.

59 En effet, «MySpace s'est allié avec plusieurs maisons de disques pour proposer un nouveau service de musique en ligne. Le réseau social a en fait créé une joint-venture baptisée MySpace Music avec Sony BMG, Universal et Warner. EMI dont la présence avait été évoquée au mois de février s'est finalement retiré; la major a décidé de soutenir le service Come With Music de Nokia. Dans les mois prochains, la plateforme MySpace Music proposera donc plusieurs formes d'écoute : du téléchargement de $\mathrm{MP}_{3}$ sans DRM, du streaming avec coupures publicitaires ou bien encore des sonneries pour mobiles. Une partie des revenus générés seront reversés aux majors en fonction de leur participation. Les artistes ou apprentis artistes disposant de leur espace sur MySpace pourront profiter de cette plateforme pour distribuer ou vendre leurs morceaux, mais aussi pourquoi pas des places de concert ou des papiers peints pour le PC. Revendiquant 30 millions de visiteurs et 5 millions d'artistes présents sur ses pages, MySpace par ces différents modes d'écoute concurrencera à la fois les sites de streaming comme Deezer ou SpiralFrog, mais aussi des plateformes de vente par téléchargement comme iTunes, nouveau numéro un de la musique aux Etats-Unis.» : http://www.cnetfrance.fr/news/myspace-et -trois-grandes-majors-musicales-s-associent-pour-contrer-itunes-39380210.htm. 
décalage ${ }^{60}$. Ce procédé de composition demande une extrême concentration à l'interprète qui pratique une sorte de double-entendre («l'entendre entendre» comme dirait Peter Szendy) ${ }^{61}$, voire de triple-entendre dans la mesure où le procédé de phasing crée acoustiquement des sous-mélodies ou des effets d'écho que le matériau musical initial ne contenait pas. Cette dernière synchronicité argumentative nous a permis de rejoindre, même succinctement, le musical proprement dit, auquel nous revenons de façon rassurante car nous y trouvons bien souvent les clés de notre compréhension du monde.

\section{Amití́(s)...}

Le partage entre amis de ce qui circule en sous-main est devenu un des signes de cette socialité virtuelle, de ses codes et de ses façons d'être. Le mot même d'ami redevenu à la mode dans les bouches de cette jeune génération semble garder un petit côté désuet et suranné ou n'être que superficiel en raison justement de son omniprésence sur la toile (réseaux sociaux, communautés...) et sa "gestion sociale» pour le moins expéditive : on ajoute ou retranche à son gré les membres de sa liste d'amis (comme on le fait avec les musiques téléchargées), avec virtuosité et sans aucun état d'âme. Cependant, et si l'on veut bien se rappeler quelques mots d'Hannah Arendt, on pourra, encore, y chercher la marque intemporelle du "vivre ensemble» que chaque époque et chaque être décline temporellement, comme un funambule. De façon précaire en expérimentant l'équilibre :

Nous avons coutume aujourd'hui de ne voir dans l'amitié qu'un phénomène de l'intimité, où les amis s'ouvrent leur âme sans tenir compte du monde et de ses exigences. Rousseau, et non Lessing, est le meilleur représentant de cette conception conforme à l'aliénation de l'individu moderne qui ne peut se révéler vraiment qu'à l'écart de toute vie publique, dans l'intimité et le face à face. Ainsi nous est-il difficile de comprendre l'importance politique de l'amitié. Lorsque, par exemple, nous lisons chez Aristote que la philia, l'amitié entre citoyens, est l'une des conditions fondamentales du bien-être commun, nous avons tendance à croire qu'il parle seulement de l'absence de factions et de guerre civile au sein de la cité. Mais pour les Grecs, l'essence de l'amitié consistait dans le discours. (...) Le dialogue (à la différence des conversations intimes où les âmes individuelles parlent d'elles-mêmes), si imprégné qu'il puisse être du plaisir pris à la présence de l'ami, se soucie du monde commun, qui reste "inhumain" en un sens très littéral, tant que des hommes n'en débattent pas constamment. Car le monde n'est pas humain pour avoir été fait par des hommes, et il ne devient pas humain parce que la voix humaine y résonne, mais seulement lorsqu'il est devenu objet de dialogue ${ }^{62}$.

60 Précisons que cette sommaire définition ne prétend être que métaphorique. D’autre part, Steve Reich s'est lui-même rendu compte des limites de cette technique initiale et l'a fait évoluer vers davantage de complexité compositionnelle et structurelle dès 1967 (Violon phase).

61 Szendy 2001, p. 167-170.

62 Arendt 1974, p. 34. 


\section{RÉFÉRENCES}

Abbate, Carolyn. 2004. "Musicologie, politiques culturelles et identité sexuelle», Musiques. Une Encyclopédie pour le XXI siècle, «2, Les Savoirs Musicaux», sous la dir. de Jean-Jacques Nattiez, 822-830. Arles-Paris : Actes Sud/Cité de la musique.

Arendt, Hannah. 1974. Vies politiques. Paris : Gallimard.

Bollack, Jean. 1997. La Grèce de personne. Les mots sous le mythe. Paris : Seuil.

Bull, Michael. 2005. "No dead air! The iPod and the culture of mobile listening». Leisure Studies, vol. 24, $\mathrm{n}^{\circ} 4: 343-355$. Routledge/Taylor \& Francis Group. (disponible via http://www.informaworld.com).

Cauquelin, Anne. 2010. À l'angle des mondes possibles. Paris : Presses Universitaires de France.

Charles, Daniel. 1998. «Pour une poétique de la multiplicité», Musiques nomades, 118-127. Paris : Kimé.

Coste, Claude. "Comment vivre ensemble de Roland Barthes", Recherches \& Travaux, 72 2008, [En ligne], mis en ligne le 15 décembre 2009. URL : http://recherchestravaux.revues.org/index107.html. (Consulté le 18 avril 2011).

Esclapez, Christine. 2003. "L'interprétation musicologique. Une dialectique entre l'utopie, l'imaginaire et la tradition? ». Imaginaire et Utopies du XXI siècle, 61-80. Paris : Klincksieck.

-2007. La musique comme parole des corps. Boris de Schloezer, André Souris et André Boucourechliev, préface de Daniel Charles. Paris : L'Harmattan.

Guibert, Gérôme. "Musique et économie plurielle. Du rôle des logiques économiques dans l'élaboration de la musique» disponible via http:// www.cdmc.asso.fr/fr/ressources/conferences/enregistrements/musique _globalisation.

Lyotard, Jean-François. 1979. La condition postmoderne. Paris : Les Éditions de Minuit.

Meschonnic, Henri. 2009. Pour sortir du postmoderne. Paris : Klincksieck.

Michon, Pascal. 2007. Les rythmes du politique. Démocratie et capitalisme mondialisé. Paris : Les Prairies ordinaires,

Millette, Mélanie. 2009. «Usages contributifs sur internet : le podcasting indépendant et le sens de son style». Mémoire de maîtrise en communication, Université du Québec à Montréal.

Pasquier, Renaud. 2007. «La valeur de l'œuvre littéraire.» disponible via http:// www.fabula.org (consulté le 7 avril 2010).

Pessoa, Fernando. 1990. Fragments d'un voyage immobile, précédé d'un essai d'Octavio PAZ. Paris : Petite Bibliothèque Rivages.

Polanyi, Karl. [1944] 1983. La Grande transformation. Paris : Gallimard.

Rastier, François. 2001. Arts et sciences du texte. Paris : Presses Universitaires de France.

Schloezer de, Boris. [1947]1979. Introduction à Jean-Sébastien Bach. Essai d'esthétique musicale. Paris: Gallimard.

Szendy, Peter. 2001. Écoute. Une histoire de nos oreilles, précédé de Ascoltando par Jean-Luc Nancy. Paris : Les Éditions de Minuit. 
Thouard, Denis. Mai 2003. "L'enjeu de la philologie». Critique. L'art de lire de Jean Bollack, $n^{\circ} 672: 346-359$. Paris : Revue générale des publications françaises et étrangères.

Vandendorpe, Christian. 1999. Du papyrus à l'hypertexte. Essai sur les mutations du texte et de la lecture. Paris: La Découverte.

Yourcenar, Marguerite.[1958] 1974. Mémoires d'Hadrien. Paris : Gallimard.

\section{RÉFÉRENCES WEBOGRAPHIQUES}

Mélanie Millette / Page personnelle : http://grm.uqam.ca/?q=millette

Rapport de Jean-Michel Fourgous, député des Yvelines «Réussir l'école numérique», 15 février 2010 disponible via http://www.rslnmag.fr/Content/ Files/fc/file/rapport-fourgous-chatel-TICE.pdf

\section{RÉSUMÉ}

Dans son article «L'enjeu de la philologie», Denis Thouard ausculte ce qu'il appelle la culture post-livresque, c'est-à-dire notre rapport actuel à l'écrit qui se concrétise par une intimité de plus en plus grande avec l'hypertexte.

C'est à partir de ce constat que nous chercherons à ausculter les conditions actuelles de diffusion, principalement caractérisées par le principe de la baladodiffusion (ou podcasting) qui interroge directement la question économique mais aussi esthétique de la réception musicale. Loin de dresser un bilan totalement négatif de ces nouvelles conduites d'écoute qui ont tendance à soumettre la qualité (choix de l'interprétation par exemple) à la quantité des téléchargements musicaux effectués, nous en discuterons de la façon la plus ouverte et la plus utopique possible. Nous ferons l'hypothèse qu'esthétiquement, il y a là, quelque chose d'extrêmement intéressant qui est en train de repousser imperceptiblement les limites de la sphère économique et même politique. Face à cette envie portative, immédiate, relative et désordonnée de musiques plurielles, il y a aussi un renversement des valeurs qui sont les nôtres : la quantité de musiques écoutées n'est plus nécessairement synonyme d'appauvrissement, d'inculture ou d'asservissement aux logiques de l'économie de marché, mais procède aussi de ce désir de découvrir et de tendre l'oreille. Désir où le total désordre stylistique et chronologique permet de refonder cette continuité historique mais aussi anthropologique qui semblait avoir déserté la seconde moitié du XXe siècle.

\section{ABSTRACT}

In his publication entitled L'enjeu de la philologie, Denis Thouard examines our relationship to the written text embodied in what he calls the post-written culture, characterised by our increasing familiarity with hypertext. It is the starting point of our study of the actual conditions of diffusion, especially in terms of podcasting and of the economical and aesthetic issues in music reception it raises. Far from portraying only negative aspects of these new listening conditions, which make musical quality (choice of performance, for instance) subordinate to the uploaded music available, this article wishes to discuss the topic with an open and positive approach. It is hypothesised that at the aesthetic level, these conditions are very much interesting in that they seem to limit unnoticeably the influence of the political and economical spheres. Following 
this desire for portable, immediate, relative, disorganized, and plural musics, one can observe an inversion of our values: the quantity of music listened to is no longer a sign of impoverishment, ignorance or of subordination to the market's economical laws, but can also be an expression of a desire for discovery and to strain an ear. A desire in which the stylistic and chronologic disorder can help found anew a historical and even anthropological continuity which seemed to have disappeared in the second half of the zoth century. 